\title{
Analysis of Opportunism Behavior in Construction Engineering Projects Based on Evolutionary Game Theory
}

\author{
Xu Ruhang ${ }^{1, a}$, Li Lingwenying ${ }^{1, b}$, Tang Qingfeng ${ }^{2, c}$, Lv Meijing ${ }^{3, d}$ \\ ${ }^{1}$ North China Electric Power University, No.2 Beinong Road, Changping District, Beijing, China \\ ${ }^{2}$ State grid JiBei electric power economic research institute, south lane of Dizang temple No.1, \\ Fuxingmen avenue, Xicheng District, Beijing, China \\ ${ }^{3}$ Network Information Center University of Finance and Economics, 39 South College Road, Haidian \\ District, Beijing, China \\ asdxu8888@foxmail.com, b610020488@qq.com, 'tangqingfeng@163.com, drvmeijing@cufe.edu.cn
}

Keywords: Opportunism behavior, Construction engineering projects, Evolutionary game theory Abstract. Opportunism behavior is an important factor that affects the outcome of a construction engineering project. But this factor is often ignored in the field. This paper studied the effect of opportunism behavior on construction engineering projects based on evolutionary theory. Firstly, this paper explained the mechanism and consequences of opportunism behavior in the perspective of evolutionary game theory. Secondly, this paper building a model to describe the agent behavior in single population. The result shows that opportunism behavior may spread in the agents and rule out the hornist agents. This paper suggests that the opportunism behavior of agents should be properly treat so as to guarantee the outcome of the engineering projects.

\section{Introduction}

Opportunism behavior is an important factor that affects the outcome of a construction engineering project. Tanga et al.[1] point out that there exist phenomenon that some managers are unprofessional in many construction projects, which may lead to failure for them to control the progress effectively. For example, some owners require unreasonable constructive acceleration. Schedule delays may arise in the projects due to settlement price differences, materials or engineering unqualified acceptance, the low quality of workers and many other reasons. For construction projects, opportunism behavior in contract management, geological exploration, wage payment and materials supply, will all cause a significant impact on progress. Dong ga[2] point out that the project bidding system is not strictly implemented so that some projects are suspected of backroom deals and some involved in commercial bribery, affecting the project performance. Benevolenskiy et al.[3] think that in many existing construction projects, there is the problem of not professional project managers, which would have an inverse effect on the quality of projects.

Many studies shows that opportunism behavior is an important factor that affects many construction project targets. Lu et al.[4] pointed out that the contractual and relational governances are important to improve project performance, and these two factors function as complements rather than substitutes. The contractual governance is more effective in improving performance while relational governance is more powerful in restricting opportunism. Kadefors el al.[5] pointed out that contractual incentives and close monitoring of contractor performance may induce opportunism and start vicious circles, and it is argued that a higher level of trust would improve project performance.

\section{Opportunism behavior in the perspective of evolutionary game}

Different from classical game theory, evolutionary game assumes that the game participants have limited rationality. People involved in the game are not to determine their strategy by rational analysis before the game but to change strategies through the so-called replication or learning. The change in the proportion of individuals adopting different strategies in population is achieved through their descendant replication. If one strategy can make it produce more offspring, the proportion of 
individuals using this strategy will increase in the population. This changing process is dynamic, and in order to find its steady state we need to research by means of its own dynamic development mechanism.

Based on such dynamic mechanism, more realistic theoretical assumptions can be conducted. For example, in the classic game theory, hybrid strategy is a concept so away from the common knowledge of life that we can't imagine a person will randomly select one from the existing policy set in some probability when making decisions. But in evolutionary game theory, we can assume that each individual only uses pure strategy.

First, we analyze the dynamic equilibrium formation mechanism of population strategy from the perspective of mathematical analysis. Suppose a group composed of a plurality of individuals contains $n$ types of pure strategy, that is $1 \ldots n$. At the same time, assume that observed time is continuous and every individual is using one of the strategies at every moment. For strategy $i, s_{i}$ represents for the proportion of people using this strategy in the population. Accordingly, there is a vector $s=\left(s_{1}, s_{2}, \ldots, s_{k}\right)$, called the population state vector. The return $F(i \mid s)$ obtained by an individual using strategy $i$ per unit time is a function of $i$ and $s$, which is also referred to as the adaptability of some strategy. This indicates that the return that an individual adopting a strategy can get during gaming with other individuals is related to the proportion of each strategy in population.

The basic idea: the more adaptability a strategy has, the more likely it to be adopted in the future. The more applicable a strategy is, the higher its proportion in the population will be. Based on evolutionary game theory, the definition of equilibrium is: if the function F(i|s) of every pure strategy $i$ is equal in state $p$, the state $p \in K$ is called a steady state.

The perspective of evolutionary game provides a framework for simulating the game process, in which quantization hypothesis can be conducted for the nature of the process and opportunism behavior. The basic idea of this framework is shown as follows:

We assume that the regulatory process takes place between several population, $p \in$ population , $i \in \mathbb{Z}$ 。population is a collection of all parties in our study. Then there can be two population. $p_{1}$ is population including all possible parties with regulators behavioral characteristics while $p_{2}$ represent for all possible parties with regulated behavioral characteristics like contractor. In practice, which population a specific party belongs to should be determined by the particular agency relationship we need to study. $n_{i}$ represents for the total number of individuals in population $p_{i}$.

Assume a time-dependent process, $\operatorname{process}(S, t)$, where $t \in T$ represents for the measurement of time and $S$ is the state we are concerned. For example, for regulatory issues $S$ may be the total number of people adopting opportunism strategies. $S_{i} \in$ strategy $_{i}\left(p_{i} \in\right.$ population $)$ is the current strategy used by an individual of a population and strategy $_{i}$ is a strategy collection of a particular population.

$S$ is likely to be a variable relevant to ni and strategy $_{i}$. Besides, $S$ may be related to time and may also be unrelated based on the nature of the process. If the time in the process is divisible, it may be affected by the previous state, $S_{n} \sim F\left(\left\{S_{j}, 0 \leq j \leq j-1, j \in T\right\}\right)$. Under the framework of the process, regarding the complexity of regulatory issues brought by exogenous factors we can measure $\theta \in$ nature with multiple "external tags" of process and nature is on behalf of the external conditions that are independent of the game participants. Then according to observation of practice, we may obtain the distribution these factors obey in the process, that is $\theta_{i} \sim F_{i}(\mu, \sigma), i \in \mathbb{Z}$. Finally, according to the nature of the process, we may get a pure stable result, also probably an unstable one. Overall, steady state in engineering process may depend on the number of population, population strategy space, policy mutability, the process transformation mechanism, as well as external factors. Based on the principles of evolutionary game theory, we can look for evolutionary stable strategy in the process to analyze the state stability in this model, and thus find a regulatory approach that can bring the optimal steady state according to the model. 


\section{Opportunism behavior model in construction projects}

Next, this paper examines the evolution of the regulated (namely agents') opportunism behavior by using single population replicated dynamic equation. We assume that each individual will pairwise game in the single population consisting of agents: Whenever establishing agency relationship with the clients, these two individuals will compete with each other. Suppose there are two strategies in this population, the first strategy is not to adopt opportunism behavior while the second one is to use. The so-called opportunism behavior includes the following meaning: to secure contracts at a lower price, and then get benefits that are not provided in contracts by use of information asymmetry during the execution. On one hand, such behavior will damage the interests of the clients, and on the other hand it may harm the interests of other agents that normally participate in the competition because due to the consequent higher bid they have less chance to obtain contracts than those who adopt opportunism behavior. Suppose the game payoff matrix in the single population is shown as Figure 1.

Opportunism Behavior (O) Obey Contract (B)

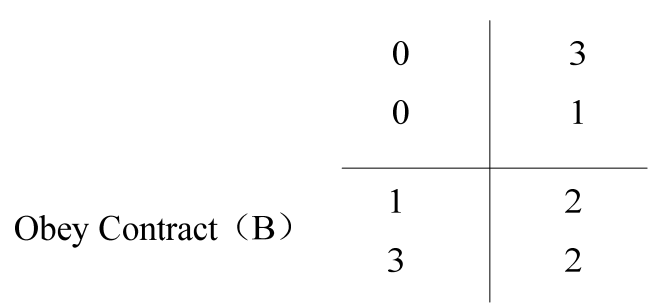

Fig.1 payoff matrix of agents in single population

When two individuals using opportunistic strategy $O$ game, their payment are both 0 because of their creating a vicious competition between each other. When an individual using opportunistic strategy games with a individual adopting compliance strategy $B$, the former will have an advantage, thence assume that the former has a greater expected return. When individuals both adopting compliance strategy game, their income is 2 . Let $x=\left(x_{1}, x_{2}\right)$, where $x_{1}$ is the ratio for the introduction of strategy $O$ and $x_{2}$ is the ratio for the introduction of strategy $B$.

Establish replicated dynamic equation for this model and the expected return of the individual using strategy $O$ can be obtained, that is:

$\pi(O, x)=0 \cdot x_{1}+3 \cdot x_{2}$

The expected return of the individual adopting strategy $B$ is:

$\pi(B, x)=1 \cdot x_{1}+2 \cdot x_{2}$

So we can draw average return of population, namely:

$\tilde{\pi}(x)=x_{1}\left(3 x_{2}\right)+x_{2}\left(x_{1}+x_{2}\right)$

$\tilde{\pi}(x)=x_{2}^{2}+4 x_{1} x_{2}$

Then replicated dynamic equations of the two population can be respectively established as:

$\dot{x}_{1}=x_{1}\left(3 x_{2}-x_{2}^{2}-4 x_{1} x_{2}\right)$

$\dot{x}_{2}=x_{2}\left(x_{1}+2 x_{2}-x_{2}^{2}-4 x_{1} x_{2}\right)$

Notice that $x_{1}+x_{2}=1$. Let $\dot{x}_{1}=0, \dot{x}_{2}=0$ and seek its stagnation point. There only one solution, that is $(1,0)$. Explanation of this solution is as follows: In equilibrium, individuals using strategy $O$ occupy the entire population. That is ultimately the entire population will adopt opportunism strategy.

\section{Conclusions}

It can be seen that the advantage of evolutionary game model is that using dynamic equation can analyze the law of opportunism behavior's appearance and development in the population. For this rule, we could affect the possible mutation strategy payment to control its ESS. Therefore, based on 
this model, it will be possible to establish efficient regulatory approaches for the opportunistic behavior in construction projects. It is suggested that opportunism behavior of agents should be properly treat so as to guarantee the outcome of the engineering projects.

\section{References}

[1] Yuanjie Tanga, Rengkui Liua, Quanxin Sun. Schedule control model for linear projects based on linear scheduling method and constraint programming [J], Automation in Construction, 2014, Vol 37 (2014), p.22.

[2] Ning Donga , Martin Fischera, Zuhair Haddadb, Raymond Levitt. A method to automate look-ahead schedule (LAS) generation for the finishing phase of construction projects [J]. Automation in Construction, Vol.35 (2013), p.157.

[3] A. Benevolenskiy, K. Roos, P. Katranuschkov, R.J. Scherer. Construction processes configuration using process patterns[J]. Advanced Engineering Informatics, Vol.26(2012), p.727.

[4] Ping Lu, Shuping Guo, Lamei Qian, Ping He, Xiaoyan Xu. The effectiveness of contractual and relational governances in construction projects in China[J]. International Journal of Project Management, Vol. 33 (2015), p.215

[5] Anna Kadefors. Trust in project relationships - inside the black box[J]. International Journal of Project Management, Vol 22 (2004), p.175 